\title{
HYPERTENSION: PATIENT ADHERENCE, Health Beliefs, Health Behaviour AND MODIFICATION.
}

\begin{abstract}
This paper presents the difficulties inherent in the management of hypertension. As patients are required to manage their hypertension by means of changing their health behaviour their own personal health beliefs come into play. Patients' health beliefs are dependent on their particular culture, their understanding of health and their reactions to the health care system. An argument is made that for health behaviour to change patients have to be seen within a system which includes themselves, their families and the health care providers. Health advice has to be appropriate for individual patients and they have to be supported by their families and the health care system
\end{abstract}

\section{KEY WORDS: HYPERTENSION, ADHERENCE, HEALTH BELIEFS, HEALTH BEHAVIOUR, MODIFICATION.}

\section{HYPERTENSION-INCIDENCE AND MANAGEMENT}

There is a high incidence of hypertension in South Africa. Out of a population of approxinately 42 million, five and a half million South Africans have blood pressures above 140/90. Of these three million are black South Africans (Steyn et al 1992).

Stroke is one of the most disrupting and alarming complications of hypertension. Rates of between $32 \%-53 \%$ are reported in Southern Africa. In addition data suggest that stroke is the second most common cause of death in Southern Africa (Lisk 1993; Matenga et al 1986). It therefore becomes essential that hypertension is controlled to prevent complications like stroke.

In 1992 guidelines were developed in South Africa for the management of hypertension (Heart Foundation Consensus Statement 1992). Persistently high blood pressure of more than $160 / 90$ or 100 is usually the level at which

\section{CORRESPONDENCE TO:}

A Stewart

Department of Physiotherapy

University of the Witwatersrand

7 York Road, Parktown 2193

Tel: (01 I ) 488-3450

Fax: (011) 488-3210

Email: 159aimee@chiron.wits.ac.za pharmacological intervention begins. For blood pressures of more than 140/90 non-pharmacological management or life-style modification should be implemented (Heart Foundation Consensus Statement 1992; JNC V 1993). Effective control of blood pressure should be achieved by the least intrusive methods possible. Non-pharmacological management or life-style modification should be "vigorously encouraged' (JNC V 1993). Non-pharmacological intervention when properly used offers many benefits at minimal risk to the patient and at little cost.

Patients should be empowered to participate in their own management. The guidelines have clearly stated educational principles which include knowledge of the disease and risk factor modification (Heart Foundation Consensus Statement 1992). Patients have to adhere to a management programme.

\section{ADHERENCE TO RISK FACTOR MODIFICATION BY HYPERTENSIVE PATIENTS}

Adherence to any kind of medical intervention in the long term, requires some form of behaviour modification. This behaviour modification may range from a simple change to one that requires complex changes. The modification is complex when considerable life-style changes are involved (Agras 1989).

Poor adherence occurs in all chronic disease regardless of the particular socio -economic level of the patient. It does tend to be much more of a problem in lower socio-economic groups (Haynes 1987). Hypertension tends to be more prevalent and also devastating in lower socio-economic groups (JNC V 1993).

Poor adherence is associated with many factors. These factors include general poor health, being unmarried, unemployed, a lower income, being a smoker, being younger, having an increased mass, complex drug regimens, side effects, cigarette smoking, high salt use, long term treatment, excessive alcohol use, perceived barriers to treatment, beliefs about the disease and social normative values (Flack et al 1996; Norman et al 1985).

Two problems exist when examining non-adherence. Firstly, current theories only partly explain non-adherence, and secondly, health-care providers seldom act according to recommendations derived from research findings (Sluijs and Knibbe 1991).

Health-care professionals tend to underestimate the frequency of adherence problems and what is involved in trying to adhere to health-care advice. They also do not understand the difficulties involved in trying to adhere to a complicated medical regimen (Sotile 1996). There are five main components of adherence behaviour. These are: 
starting treatment; keeping appointments with medical personnel; taking medication correctly; making life-style changes and avoiding heath-risk behaviours (Agras 1989).

If life-style modification is to be considered an important part of the management of hypertensives, healthcare practitioners should understand how patients perceive their own health and its management (Morisky et al 1986; JNC $\mathrm{V}$ 1993). Adherence needs to be seen in the light of total health promotion rather than just a narrow adherence to a particular medical regimen (Spilker 1996).

Blaming patients for treatment difficulties ignores the shared responsibilities of others, like the family and medical personnel (Sotile, 1996 ). Patients who only receive routine care are more than twice as likely to drop out of treatment than those who receive routine care and periodic visits from health-care practitioners. The addition of some sort of family support whether it is structured or informal also increases adherence (Sotile 1996; Jensen and Lorish 1994).

Time may well be an important factor in understanding adherence behaviour. "The discounting effects of future events" may affect a patient's willingness to adhere (Brown and Segal 1996). By this is meant that the benefits of adherence which are only felt far in the future do not necessarily motivate patients. So the effect of time needs to be considered when developing adherence models.

\section{HEALTH}

Prior to considering health behaviour some consideration needs to be given to what patients understand by health. In a study done in the UK patients described good health as being able to function, not having any symptoms, the absence of illness, feelings of well-being and of being fit (De La Paz 1992). Hypertensive patients in Alexandra described feeling well when they felt strong and could get up in the morning and do their work. When they were not feeling wel] they felt weak and did not want to get up (Stewart et al 1999). In both studies patients interpreted health in a functiona] way, as being able to cope, feeling fit and being symptom free.
Although there are varied meanings of health it is generally recognised as being one end of a continuum, the other end being disease. Thus health can be a comparative term. So at the one end of the continuum patients experience wellness and the other end of the continuum patients perceive illness. Feinstein used the term illness to describe what the patient showed or felt as a result of an underlying disease (in Fabrega 1979). Illness can then be seen in certain types of behaviour. Certain symptoms will result in the patient not being able to continue his usual activities, he may elicit sympathy from family or it may result in the person seeking medical help. So illness can be seen as the outward manifestation of a disease in its broadest behavioural sense. Although patients may have a chronic disease they may still be able to feel healthy or well (Lorig, 1996).

People can also be thought of as more or less healthy and can be judged against a standard or ideal of health. So health can also be a relative term. People will experience their health as wellness (Jensen et al 1997). Health was defined by the WHO in 1947, as "a state of complete physical, mental and social well-being and not merely the absence of disease and infirmity".

The standards or ideals of health have been encapsulated in four models of health. These are the eudaimonistic, adaptive, role performance and clinical models. The eudaimonistic model was originally proposed by the ancient Greeks and has its modern counterpart in Maslow's idea of personality (1966). Here health means the highest aspirations of an individual, that is self actualisation or in this context "exuberant well-being". In the adaptive model disease is seen as the person's inability to interact with his social or physical environment. Role performance is seen as the common sense model of health. People measure how healthy they are relative to how well they can perform their daily activities or their different roles. The clinical model sees health as being the absence of disease (Smith 1981). This model is very limited in that it implies that patients with chronic disease cannot be healthy and cannot have a good quality of life.

The clinical model sees individuals as physiological systems; the role performance model sees individuals as parts of a social fabric. The adaptive model considers individuals as flexible effective participants in the environment and the eudaimonistic model as the ideal of the civilized cultured person with the capacity for continuous growth (Smith 1981).

\section{HEALTH BELIEFS AND HEALTH BEHAVIOUR}

As described above adherence issues can be problematic as there are many variables which have been identified which relate to adherence. This is particularly so when considering situations in which patients have to adhere to life-style changes such as non pharmacological intervention in hypertension. Health behaviour is the crucial dependent variable in any health educational programme.

In order to address these issues various models of health behaviour have been developed and refined over the years. These models help to explain why patients behave in certain ways and provide a basis for health education and promotion interventions (Glanz et al 1997). Three models will be discussed here.

The health belief model as described by Glanz et al (1997) is one of the oldest and most resilient of the models. This model hypothesises that behaviour is dependent on two variables, namely - the value that a patient places on a particular goal and the likelihood that a given action will achieve a given goal. Patients will consider their susceptibility to a particular disease and will evaluate the severity. Having done that they will then make the judgement as to whether the benefit to be gained from a particular behaviour is worth the cost.

In basing an intervention on the Health Belief Model the concepts of the model can be used in the following way. Patients' susceptibility to the risk of serious health consequences has to be personalised. Patients have to understand very clearly what their own risk of serious consequences of their disease is. The benefits of improved health behaviour again need to be personalized. The difficulties or barriers associated with changed health behaviour need to 
be identified and strategies put in place to minimise the barriers. Patients also have to have confidence in their ability to change their behaviour (Glantz et al 1997). This model does not take into account the influences of the patient's family and social system (Dracup and Meleis 1982).

The stages of change model describes how patients undergo a process of change in order to bring about more beneficial health behaviour (Prochaska et al 1992). This model helps the health care practitioner to match his intervention to the patient's frame of reference as the patient attempts to modify his behaviour. In the precontemplation stage patients are mired in inactivity and do not intend making any behavioural change. Typical reasons are, lack of knowledge, knowledge gaps and demoralization. Patients will only advance to the contemplation stage if they realise that the pros of changing outweigh the cons. However patients in this stage will not advance to the preparation stage unless reasons not to change decrease. Patients in the preparation stage are ready to take immediate action to change their health behaviour. They believe the benefits of behavioural change will outweigh the costs but need to understand that they may need more than one attempt at behaviour change. During the action stage patients need to place most of their physical and emotional energies into achieving the desired behaviour change. Once they move into the maintenance stage patients' confidence increases and temptation to return to unhealthy habits decreases. Few patients progress to the termination stage where they have total confidence that they will not revert back to their old habits. Most patients have to continue with maintenance efforts. Health promotion needs to accommodate these stages.

As patients progress through the six stages of the above model they go through a process of change. This process of change involves a number of both overt and covert activities as patients move from stage to stage of health behaviour modification (Glanz et al 1997).

Both the health belief model and the stages of change model include self-efficacy as part of the model. Self-efficacy is the ability of an individual to perform a particular behaviour. (Banduras 1977). Self-efficacy is an important prerequisite for behavioural change.

The stages of change model and the health belief model describe a patient's reactions to situations in which he finds himself as well as the analytical processes he goes through when attempting to change behaviour. Neither of these models puts the patient into a social and family context nor do they show how the patient can be affected by his interactions within a social and family context.

A systemic approach has been suggested to overcome criticisms of the previous models. The systemic approach suggests patients, their families and health-care practitioners have a shared responsibility to respond to medical intervention. The focus is on the relationships between the patient and health-care practitioner, between the patient and his or her family and the family and the health-care practitioner (Doherty and Baird 1983 ).

Harkaway and Madsen (1989) present a model that considers the healthcare practitioner as an active participant in the family and treatment systems. They suggest that treatment problems occur in the context of the above triad and not as a result of one of the individuals involved. They emphasise patients' beliefs about the problem; beliefs about the treatment of the problem; beliefs about the role of the health-care practitioner and beliefs about the role of the family.

Beliefs about the problem will influence how patients view the problem. If for example patients and their families consider "hypertension" as a disease involving the nerves it is unlikely that they will consider dietary restrictions or increasing exercise output as relevant (Heurtin- Roberts and Reisin 1992). Other culturally held beliefs will also influence patients' and their families' willingness to adhere to health advice (Heggenhougen and Shore 1986; Atuado 1985; Hugo 1992 ). In addition to the beliefs held by the patient and the family unit the health-care practitioner may have beliefs about the problem.
The attitude of health-care practitioners will be influenced by previous experiences and expectations. There may be conflicting attitudes of respect and mistrust. Cultural differences between patient and health-care practitioners lead to misunderstandings of the role of the health-care practitioner (de Villiers 1991; Hugo 1992; Atuado 1985). In addition there may be an authoritarian health-care provider and a passive patient and family. There may not be equal participation from all individuals within the system.

The beliefs of the family also need to be considered. If a family's belief about a disease and its role as a support system is not convergent with that of the patient and health-care practitioner difficulties in modifying health behaviour will result. The family may resent the perceived intrusion of the health-care practitioner as it tries to cope with a family member's ilness or it may abdicate all responsibility to the health-care practitioner.

For health behaviour modification to occur patients' health beliefs need to be considered and close relationships between the triad of patient, family and health-care practitioner need to be developed.

\section{BARRIERS TO HEALTH BEHAVIOUR}

A simple model which describes the barriers to health behaviour modification has been suggested by Bartlett (1982). He divides factors which can influence patients' behaviour into four categories of barriers which will interfere with the patient's ability to adhere to advice. They are individual factors, social factors, environmental factors and the medical regimen.

Individual factors include patients' knowledge, motivation, fears, attitudes, denial and self-control. Social factors include the influences of family, peers, employers, teachers and health-care providers. Poor finances, transport, inaccessible clinics and long waiting time at clinics constitute the environmental barriers. The complexity and side effects of the medical regimen can also act as barriers to adherence. A complex relationship exists between the barriers with one being able to influence the other. In planning adherence programmes the health professional should take note of 
these barriers (Bartlett 1982; Richardson et al 1993).

One of the most crucial individual barriers to changing health behaviour is patients' lack of knowledge about their disease, what its consequences are and how to look after themselves. Prochaska et al (1992) have gone so far as to conclude that health behaviour change in hypertensive patients cannot take place without patients understanding hypertension, its risk factors, its management and the consequences if left untreated.

Hypertensive patients in the public health-care system in South Africa do not understand the causes of the disease, particularly the lack of exercise as a risk factor. They have some idea of a healthy diet and restricting salt ingestion (Stewart et al 1999; Stewart et al 2000). Those with higher educational levels, those who spoke English or Afrikaans and had a better quality of life and sex life were more knowledgeable about their disease (Stewart et al 2000). Williams et al (1998) showed a similar level of understanding in hypertensive and diabetic patients with low literacy levels. Between $35 \%-90 \%$ of patients do not understand the information that is given to them (Prochaska et al 1992).

The consequences of uncontrolled hypertension were not well understood in the group of patients studied by Stewart et al (2000). Kattapong et al (1998) demonstrated that Hispanic women were significantly less likely to consider hypertension as a risk factor for stroke than non-Hispanic women. Both groups were found to have poor understanding of the risk factors for stroke.

Influences of society can be a profound social barrier. Influences of culture, societal norms and family can affect patients' health behaviour. When examining the adherence to medical advice of patients with chronic disease the effects of culture cannot be underestimated. (Heggenhougen and Shore 1986).

Health and illness need to be seen according to the standards of adaptation which are employed by different cultural groups. Traditional societies regard health as a state of balance or equilibrium and traditional medicine achieves this balance. Health is seen in traditional societies as encompassing the physical, mental, social, moral and spiritual wellbeing of individuals (Ataudo 1985).

Comparative studies of blood pressure levels have shown that in societies with a coherent value system which remains relatively unchallenged over generations blood pressure values are low but that migration to societies where the value systems are different, high levels of blood pressure occur (Heggenhougen and Shore 1986). Although many practices which are linked to ill-health are a matter of personal choice they are often condoned within a particular culture. Examples are, alcohol consumption, which is socially accepted in most cultures (with Muslims being notable exceptions) and dietary practices.

Effective use of orthodox medicine depends in part on the congruence of patients and health-care practitioner's beliefs about illness and treatment. Variations in underlying knowledge systems impede even willing adherence on the part of culturally divergent groups. This is because information is not understood when it is first communicated or it is not accurately recalled. What one understands is a function of how well the information fits one's existing knowledge (Stefferson and Colker 1982).

The relationship between the healthcare provider and the patient can be a social barrier to adherence. This is often between people of different power and different cultures resulting in a huge cultural gap between patients and medical practitioners. Traditional medicine pays attention to attitudes and feelings whereas there is a lack of communication in allopathic medicine (Heggenhougen and Shore 1986).

In South Africa medical encounters often occur in a transcultural context. When the understanding of the patient and the health-care practitioner differs, problems tend to occur in the clinical setting and this reduces the effectiveness of the encounter. This has negative implications for the management of the patient (De Villiers 1991). Many people in South Africa find it difficult to experience healing within a Western healthcare system. This is because in such a system they experience more about disease and curing than about illness and healing. A traditional healer connects a patient's symbolic reasoning to his body and what he experiences in his body (Hugo 1992).

Environmental barriers become enormously important for patients from lower socio-economic classes who have to use the public health system. The cost of transport to the hospital; cost of treatment; loss of work time with the resultant loss of earnings and the cost of a prudent diet make adherence for hypertensive patients difficult (Stewart et al 1999). In addition the long waiting times which can be anything up to a five hour wait to see the doctor for 10 minutes and then a further five hour wait at the pharmacist for their medication prove to be significant environmental barriers for patients (Stewart et al 1999).

Patients use a variety of criteria to determine the value of medication and this may become a barrier to adherence to medical regimens. They may place an equal or greater value on competing non-clinical outcomes. Physical, economic, psychological and social factors influence the use of medication. Patients often take less drugs than required, due to their experiences with side effects and their symptoms (Wallenius et al 1995).

\section{HEALTH BEHAVIOUR MODIFICATION}

Sotile (1996) has suggested that health behaviour modification programmes should be structured such that patients' participation is facilitated. He suggests that such programmes should only last for three to six months. It is easier for patients to commit for this length of time and they are then less likely to drop out. Patients need to leave these programmes with enhanced self esteem. This is crucial in promoting self-efficacy, which is the key to lasting changes in health behaviour.

The key element in promoting a wide range of risk factor changes is educating the patient and the family (Sotile 1996). Inclusion of the family in an education programme supports the patients' efforts to modify health behaviour.

There are only a few studies in the health education literature which show alterations in health behaviour rather than just a change in knowledge or attitudes as a result of an educational 
intervention. Education alone does not change health behaviour although knowledge is a prerequisite for a behaviour modification programme (Cohen and Cohen 1987). So health promotion or patient education is a planned learning experience using a combination of methods such as teaching, counseling and behaviour modification which will influence patients' knowledge and health behaviour (Sotile 1996; Hill 1989). Patient education and patient counseling involve an interactive process which assists patients to participate actively in their health care (Bartlett 1985). Education should be individualised, the patient should have rapid feedback, there should be a clear understanding of what is required, the patient should be motivated and there should be congruence between the patient's and health-care practitioner's goals.

Different types of people need and respond favourably to different kinds of interventions. Age, education literacy levels and socio-economic variables are the most important factors in predicting receptivity to health education. Patients with lower educational levels have been shown to respond to individualised clarification of instructions and the support of a family member, whereas those with a higher level of education respond to small group interactive meetings (Morisky et al 1986; Morisky et al 1985). The educational programme should include common sense strategies for better managing the life-style changes required for non-pharmacological intervention of hypertension (Morisky et al 1985; Hill 1989; Sotile 1996).

As with physical diagnosis and medical care, patient education should be based on careful assessment, a correct diagnosis and an individualised plan of health behaviour modification. Healthcare practitioners must be able to identify the specific needs of patients for information, skills, support and reinforcement. They must meet these needs with a combination of educational strategies adapting approaches as necessary over time. Health-care practitioners need to determine the quantity and quality of information, interactions and procedures that reinforce appropriate behaviour.

It becomes increasingly evident that in order for health behaviour modification programmes to be effective they have to be patient centered and have as their foundation an unambiguous educational component to improve patients' knowledge about their disease and its management. This needs to be enhanced by creating close relationships between patients, their families and health-care providers within a supportive environment.

Health education influences patients' behaviour through changes in knowledge, attitudes, beliefs, perceptions and social support, through self-reinforcement and skill development and through changes in professional behaviour towards patients (Hill 1989). The strategies for patient education include - identifying their existing knowledge, attitudes and beliefs and experiences; educating about the condition and treatment; tailoring the regimen to the patient; providing reinforcement; promoting social and family support and collaborating with other professionals as required (Hill 1989; Sotile 1996; Bartlett 1982).

\section{CONCLUSION}

If the non-pharmacological management of hypertensive patients is to be taken seriously patients need to be evaluated and given the necessary support in order to make it possible for them to modify their health behaviour. Patients need to be appropriately supported by both their families and their health care providers in order to modify their health.

\section{REFERENCES}

Agras W S 1989 Understanding Compliance with the Medical Regimen: The Scope of the Problem and a Theoretical Perspective Arthritis Care and Research 2: S2-S7

Ataudo E S 1985 Traditional medicine and biopsychosocial fulfillment. African Health Social Science Medicine 12: 1345-1347

Bandura A 1997 Social Learning Theory Englewood Cliffs NJ Prentice Hall

Bartlett E E 1982 Behavioural diagnosis: a practical approach to patient education. Patient Counseling and Health Education 4: 29-35

Bartlett E E 1985 Editorial Patient Education and Counseling 7: 323-324

Brown C, Segal R 1996 Ethnic Differences in Temporal Orientation and Its Implications for
Hypertension Management. Journal of Health and Social Behaviour 37: 350-36I

Cohen C I, Cohen E J 1987 Sounding Board: Health education: panacea, pernicious or pointless New England Journal of Medicine 299: 718-720

De la Paz R 1992 Why me why now video recording BBC for the Open University

De Villiers S 1991 Beliefs and Behaviour in Transcultural Health Care. South African Family Practice 12: 44-49

Doherty W J and Baird M A 1983 Family therapy and family medicine: toward the primary care of families New York: Guildford Press

Dracup K, Meleis A I 1982 Compliance: An interactionist approach. Nursing Research 31:3I-36
Fabrega H 1979 The ethnography of illness. Social Science and Medicine 13A: 565-57

Flack J M, Novikov S V, Ferrario C M 1996 Benefits of adherence to anti-hypertensive drug therapy. European Heart Journal 17 (suppl A): $16-20$

Glanz K, Lewis F, Rimer B 1997 Health Behaviour and Health Education theory research and practice. 2nd Edition. Jossy Bass lnc Publishers, California

Harkaway J, Madsen W C 1989 A systematic approach to medical non-compliance: The case of chronic obesity. Family systems Medicine 7: $42-65$

Haynes R 1987 Ten year update on compliance research. Patient Education and Counselling 10: 107-174 
Heart foundation hypertension consensus symposium 1992 Management guidelines for hypertension. Southern Africa Supplement to South African Medical Journal: 1-4

Heggenhougen H, Shore L 1986 Cultural Components of Behavioural Epidemiology: Implications for Primary Health Care. Social Science Medicine 22:1235-1245

Heurtin-Roberts S, Reisin E 1992 The relation of culturally influenced models of hypertension to compliance with treatment. American Journal of Hypertension 5(11): 787-792

Hill M N 1989 Strategies for patient education. Clinical and Experimental Hypertension Theory and Practice A 11(5, 6): 1187-1201

Hugo J 1992 Experiences of sickness and healing by poor rural families in Lebowa. South African Family Practice: 258-264

Jensen G, Lorish C D 1994 Promoting patient cooperation with exercise programs. Arthritis Care and Research 7(4): 181-189

Jensen G M, Lorish C, Shepard K F 1997 Understanding patient receptivity to change: teaching for treatment adherance. In: Handbook of teaching for physical therapists, Shepard K F, Jensen G M (editors). Butterworth-Heineman: 241-269

Joint National Committee V 1993 The fifth report of the joint national committee on detection, evaluation and treatment of high blood pressure. Archives of 1nternal Medicine 153: 154-183

Kattapong V J, Longstreth W T, Kukull W A, Howard D B, Bowes J I, Wilson B E, Becker T M 1998 Stroke Risk Factor Knowledge on Hispanic and Non-Hispanic White Women in New Mexico: Implications For Targeted Prevention Strategies Health Care for Women International 19:313-325

Lisk D R 1993 Hypertension in Sierrs LeoneStroke population East African Medical Journal 70(5): 284-287

Lorig K 1996 Patient education a practical approach. 2nd edition. Thousand Oaks CA Sage

Maslow A H 1966 The psychology of science. Chicago, Henry Regnery

Matenga J, Kitai I, Levy I 1986 Strokes among black people in Harare Zimbabwe: results of computed tomography and associated risk factors. British Medical Journal 292:|649-165|

Morisky D E, Demuth N M, Field-Fass M, Green L W, Levine D M 1985 Evaluation of family health education to build social support for long term control of high blood pressure. Health Education Quarterly 12 (I): 35-50

Morisky D E, Green L W, Levine D M 1986 Concurrent and predictive validity of a selfreported measure of medication adherence. Medical Care 24(1): 67-74

Norman S A, Marconi K M, Schezel G W, Schecter M A, Stolley P D 1985 Beliefs, social normative influences, and compliance with anti-hypertensive medication. American Journal of Preventative Medicine 1(3): 10-17

Prochaska J O, DIClemente C C, Norcross J C 1992 In search of how people change: Applications to addictive behaviour. American Psychologist 47(9): 1102-1114

Richardson M A, Simons-Morton B, Annerges J F 1993 Effect of perceived barriers on compliance with anti-hypertensive medication Health. Education Quarterly 29(4): 489-503

Sluijs E M, Knibbe J J 199 I Patient compliance with exercise: different theoretical approaches to short term and long term compliance. Patient Education and Counselling 17: $191-204$

Smith J A 1981 The ideal of health: A philosophical inquiry. ANS/Nursing Education: 43-50

Sotile W M 1996 Psycho-social Interventions for Cardiopulmonary Patients a guide for health professionals. Human Kinetics Champaign Illinois *

Spaeth G L 1995 Non-compliance-not a patient problem, but rather the sign of a sick doctor-patient relationship. Opthalmic Surgery 26(3): 189-193

Spilker B 1996 Introduction in Quality of Life and Pharmacoeconomics. In: Clinical Trials, B Spilker (editor), 2nd edition. LippincourtRavin Publishers, Philadelphia

Stefferson M S, Colker L 1982 Intercultural misundertandings about health care. Social Science Medicine 16: 1949-1954

Steyn K, Fourie J, Bradshaw D 1992 The impact of chronic diseases of lifestyle and their major risk factors on mortality in South Africa. South African Medical Journal 82: 227-231

Stewart A V, Eales C J, Shepard K 1999 The Health Behaviour of Black Hypertensive patients at a Chronic Diseases Clinic in a Depressed Socio-Economic Area of Johannesburg, South Africa. The South African Journal of Physiotherapy 55(1): 11-17

Stewart A V, Eales C J, de Charmoy S 2000 The Knowledge Profile of Patients with
Hypertension. South African Journal of Physiotherapy 56(4):17-21

Wallenius S H, Vainio K K, Korhonen M J H, Hartzema A G, Enlund H K 1995 Self- initiated modification of hypertension treatment in response to perceived problems. The Annals of Pharmacology 29: 1213-1217

Williams M V, Baker D W, Parker R M, Nurss J R 1998 Relationship of functional health literacy to patients' knowledge of their chronic disease-a study of patients with hypertension and diabetes. Archives of internal Medicine 158: $166-172$ 